\title{
SHEET STRETCHING: A THEORETICAL-EXPERIMENTAL COMPARISON
}

\author{
Byoung B. Yoon, Ravi S. RaO and Noboru Kikuchi \\ Department of Mechanical Engineering and Applied Mechanics, The University of Michigan, Ann Arbor, MI \\ 48109, U.S.A.
}

(Received 3 October 1988; and in revised form 27 March 1989)

\begin{abstract}
This paper presents numerical solutions of axisymmetric sheet stretching employing an experimentally determined stress-strain curve and measured overall coefficient of friction along the punch-sheet interface. Experiments have been conducted on thin sheet metals such as mild steel, aluminum and brass with a hemispherical steel punch. Predicted values of loads, deflections, strain distributions and other relevant data are favorably compared with experimental values of these same quantities.
\end{abstract}

\section{NOTATION}

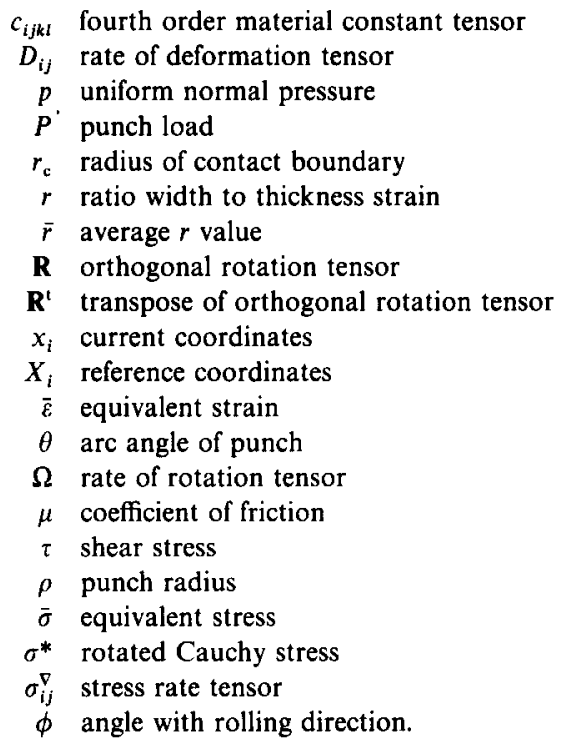

\section{INTRODUCTION}

Sheet-metal forming processes such as stretching, bending and deep drawing involve large amounts of plastic deformation. In making complex sheet-metal parts, the skill of the artisan is directly derived from the trial-and-error process. Using that process, there is a high rate of scrap from forming operations. This practice cannot survive in modern industry for several reasons: increased complexity of parts; introduction af newer materials for which no store of experience is available; greater emphasis on cost effectiveness. There is therefore a real need to replace these trial-and-error techniques by a system based on scientific understanding of the basic deformation process. The research reported here makes an attempt to address some of the practical problems using computational methods based on the finite element procedure.

Computational methods of determining process and product information of this type can be used to improve the design of the process and the quality of the product. These methods will serve as useful guidance to both producers and consumers of sheet materials. For producers, such as the steel and aluminum industries, these can be used in conjunction with experimental forming studies to evaluate the effects of different material parameters in designing more highly formable materials. For consumers, such as the automobile and aircraft industries, they can assist part designers and processing engineers in evaluating the 
severity of the forming problem, in choosing the most economical material and in choosing the sequence of operations required to fabricate the part.

One of the first comprehensive studies of cup-forming was that of Chung and Swift [1]. Their analyses focused mainly on the radial drawing process, and the materials used were considered to be isotropic. Although the radial drawing process was successfully analysed, the analysis of stretch forming over the punch head remained unsolved. Intensive analyses of sheet metal forming have been presented by many authors. Elastic-plastic [2], elastic-viscoplastic [3] or rigid-plastic [4,5] approaches have been used successfully for sheet metal deformation analysis. The rigid-plastic formulations, in which the elastic deformation is assumed to be negligible compared to the large plastic strain, have gained a wide range of applications despite the fact that the method fails to predict the stress history whenever elastic loading or unloading is encountered.

Friction is a very important parameter in metal forming processes; it has great influence on the metal flow and the necessary forming load. The extended use of mathematical and numerical analysis to simulate metal flow and predict forming forces and local pressure distributions thus requires detailed knowledge about the frictional stresses at the tool-workpiece interface. The success of theoretical or numerical simulations of metal forming processes depends on the ability to determine precisely the frictional parameters involved.

In this research, attention is focused on sheet stretching, which is mainly used to assess the stretchability of sheet metals. A circular metal blank is clamped firmly along the periphery and is stretched by a rigid hemispherical punch. The depth of the deformed sheet when it fractures may be taken as a measure of ductility. It is a complicated problem from the point of view of deformation analysis, the most notable difficulty being that of quantifying the friction over the punch head. An elastic-plastic work-hardening finite element analysis incorporating anisotropic properties of sheet metals is verified by conducting sheet stretching experiments. Preliminary results have been presented at a recent conference [6]. In this paper, results are discussed in detail for a case that uses the Coulomb friction model along the punch-sheet interface. Future work will involve detailed understanding of frictional variations along the interface using a transparent punch technique similar to that involving the drawing die developed by one of the present authors [7].

\section{EXPERIMENTAL PROCEDURES AND MATERIALS}

\section{Sheet stretching apparatus}

A rectangular die set with four posts forms the main part of the sheet stretching apparatus as shown in Fig. 1. The die set was supplied by Danley Machine Corporation. Posts and bushes were made from good quality steel, hardened, chrome plated and ground to close tolerances. This arrangement gives an exceptionally smooth hard-wearing surface that resists corrosion, lessens friction and maintains a close working fit. The die set was bolted to the base of a microprocessor-controlled Instron testing machine. The Instron is equipped with a load cell of $150 \mathrm{kN}$. Deflection can easily be monitored using the cross head speed. Load-deflection variations can be monitored and recorded using the HP $X-Y$ chart recorder.

A hemispherical punch of $50.8 \mathrm{~mm}$ radius and a die of outside diameter $206 \mathrm{~mm}$ and inside diameter $108 \mathrm{~mm}$ were made from high-carbon steel. The punch was fastened to the punch holder and the die was fastened to the die holder of the four post die set. A serrated circular groove was made on the die face for holding the blank firmly. A close-up view of the die, blank and punch arrangement is shown in Fig. 2. No lubricant was used in any of the experiments presented in this paper. The surfaces were not chemically cleaned.

\section{Material properties}

Annealed mild steel sheets of $0.762 \mathrm{~mm}$ thickness, aluminum (1100-0) sheets of $1.016 \mathrm{~mm}$ thickness and brass $(70: 30)$ sheets of $0.83 \mathrm{~mm}$ thickness were chosen as work materials. Sheet tensile specimens of gage length $165 \mathrm{~mm}$ and width $12.7 \mathrm{~mm}$ were subjected to standard tensile tests under continuous loading up to failure. The yield strength was determined using the $0.2 \%$ offset method. True stress-strain curves were approximated by 


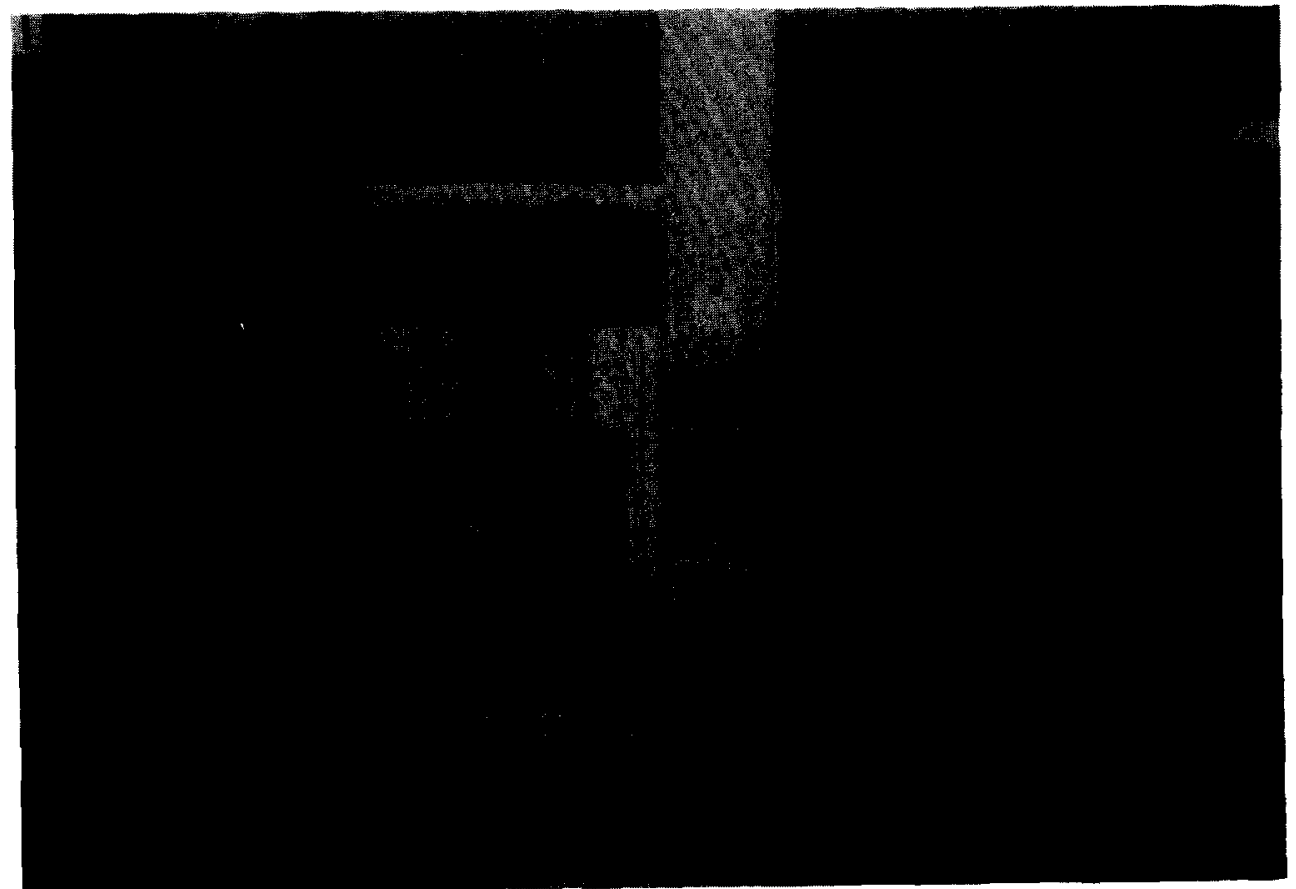

FIG. 1. Overall experimental arrangement of sheet stretching.

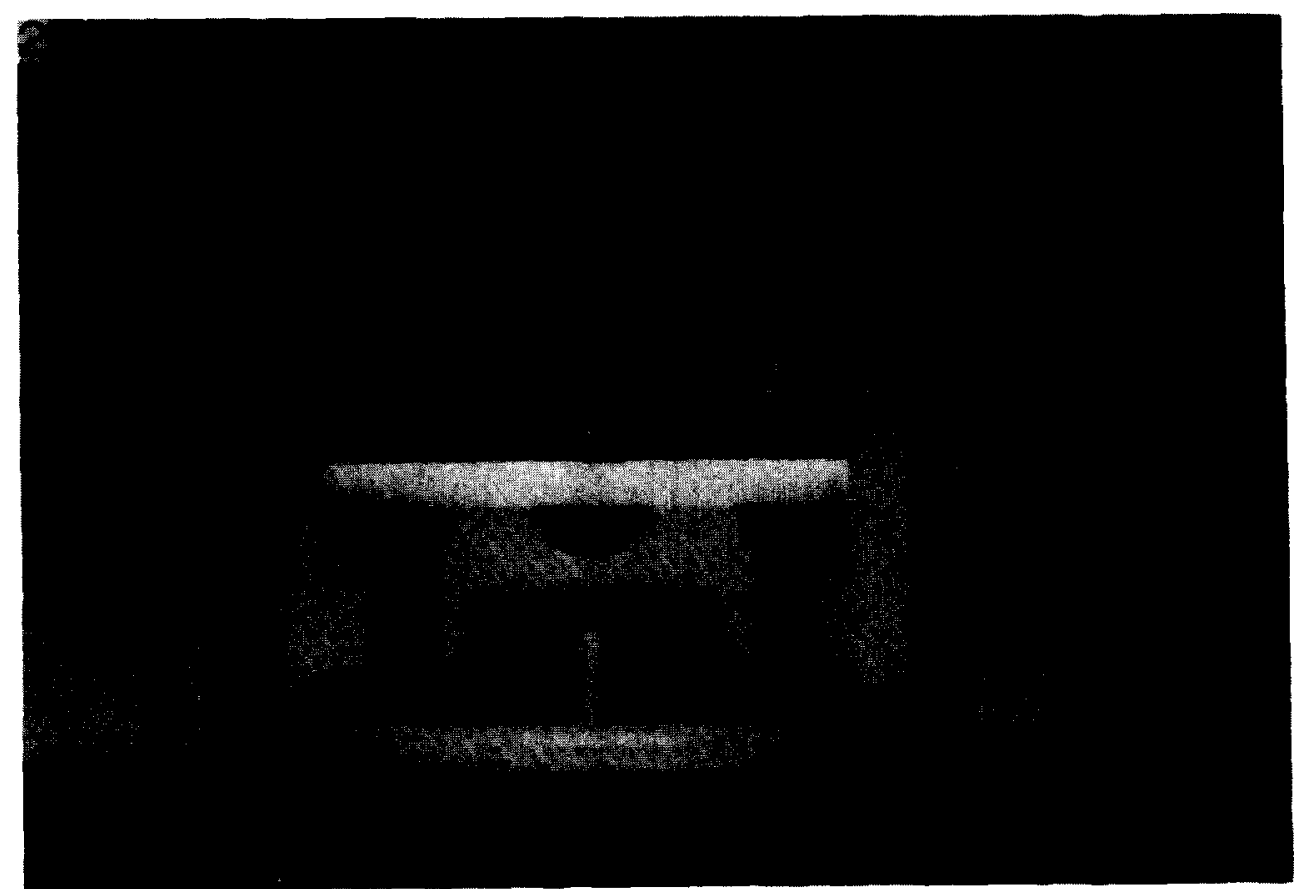

FIG. 2. Close up view of die, blank and punch arrangement. 

power law work hardening as shown below:

$$
\begin{array}{rll}
\text { mild steel: } & \bar{\sigma}=543.2 \bar{\varepsilon}^{0.2708} & (\mathrm{MPa}) \\
\text { aluminum: } & \bar{\sigma}=196.8 \bar{\varepsilon}^{0.2396} & (\mathrm{MPa}) \\
\text { brass: } & \bar{\sigma}=797.9 \bar{\varepsilon}^{0.4825} & (\mathrm{MPa}) .
\end{array}
$$

The strain ratio, usually represented by the $r$-value, is defined as the ratio of width to thickness strains. Since the thickness strain cannot be accurately measured in a thin sheet, thickness strain was deduced, using the volume constancy, from the length and width strains. The $r$ values usually vary with the test direction, $\phi$, and it is common to characterize a material by an average $r$ value, $\bar{r}$, where

$$
\bar{r}=\frac{r_{0}+2 r_{45}+r_{90}}{4}
$$

the subscript referring to the angle $\phi$ (if $\phi=0$, it means the rolling direction). From the tensile tests, $\bar{r}$ values were calculated for the materials considered as shown below:

for mild steel, $\bar{r}=1.069$

for aluminum, $\bar{r}=0.923$

for brass, $\bar{r}=0.915$.

\section{EVALUATION OF OVERALL COEFFICIENT OF FRICTION FROM EXPERIMENTS}

The model described by Ghosh [8] was adopted to evaluate the overall coefficient of friction in stretching from load-deflection plots. There are three assumptions. First, that interface pressure is uniform at any stage of deformation. Second, that Amonton's law holds: $\tau=\mu p$, where $\tau$ is the shear stress, $\mu$ is the coefficient of friction and $p$ is the normal pressure. Third, that the inflection point on the load-deflection plot corresponds to the attainment of maximum pressure. These frictional boundary conditions were used as inputs to the finite element code as described in the next section.

Figure 3 shows a deforming sheet in contact with the punch with uniform normal pressure $p$, punch load $P$, punch radius $\rho$, radius of contact boundary $r_{\mathrm{c}}$ and a frictional force $\mu p$ per unit area at the contact surface. The vertical component, $\mathrm{d} V$, of the force acting on an annular element of the sheet which subtends an angle $\mathrm{d} \theta$ at the punch center is given by

$$
\mathrm{d} V=(p \cos \theta+\mu p \sin \theta)\left(2 \pi \rho^{2} \sin \theta \mathrm{d} \theta\right) .
$$

Integration of $\mathrm{d} V$ over the entire punch contact area $\left(\theta=\theta_{\mathrm{c}}\right)$ gives the vertical force, $V$, as

$$
V=\int_{0}^{\theta_{c}} 2 \pi \rho^{2} p\left(\sin \theta \cos \theta+\mu \sin ^{2} \theta\right) \mathrm{d} \theta
$$

which equals the punch load, $P$. Integration of equation (4) leads to

$$
P=\pi \rho^{2} p\left[\sin ^{2} \theta_{\mathrm{c}}+\mu\left(\theta_{\mathrm{c}}-\sin \theta_{\mathrm{c}} \cos \theta_{\mathrm{c}}\right)\right] .
$$

Recognizing that $r_{\mathrm{c}}=\rho \sin \theta_{\mathrm{c}}$, the differentiation of $P$ with respect to $r_{\mathrm{c}}$ and substitution of $\left(\mathrm{d} p / \mathrm{d} r_{\mathrm{c}}\right)=0$ corresponding to the pressure maximum yields

$$
\left.\left(\mathrm{d} P / \mathrm{d} r_{\mathrm{c}}\right)\right]_{r_{0}}=2 \pi \rho p \sin \theta_{0}\left(1+\mu \tan \theta_{0}\right),
$$

where $r_{\mathrm{c}}=r_{0}$ and $\theta_{\mathrm{c}}=\theta_{0}$ at maximum pressure. Therefore, at $r=r_{0}$, we obtain

$$
\left[\frac{\left(\mathrm{d} P / \mathrm{d} r_{\mathrm{c}}\right)}{P}\right]_{r_{0}}=\frac{2}{\rho}\left[\frac{1+\mu \tan \theta_{0}}{\sin \theta_{0}+\mu\left\{\theta_{0} / \sin \theta_{0}\right)-\cos \theta_{0}}\right] \text {. }
$$

Replacing $\sin \theta_{0}$ by $\left(r_{0} / \rho\right)$ and $\cos \theta$ by $\left(\sqrt{\rho^{2}-r_{0}^{2}} / \rho\right)$, equation (7) would now lead to the following expression for determining $\mu$ :

$$
\mu=\frac{2-X r_{0}}{X\left\{\left(\rho^{2} / r_{0} \sin ^{-1}\left(r_{0} / \rho\right)-\sqrt{\left(\rho^{2}-r_{0}^{2}\right)}\right\}-\left[2 r_{0} / \sqrt{\left(\rho^{2}-r_{0}^{2}\right)}\right]\right.},
$$




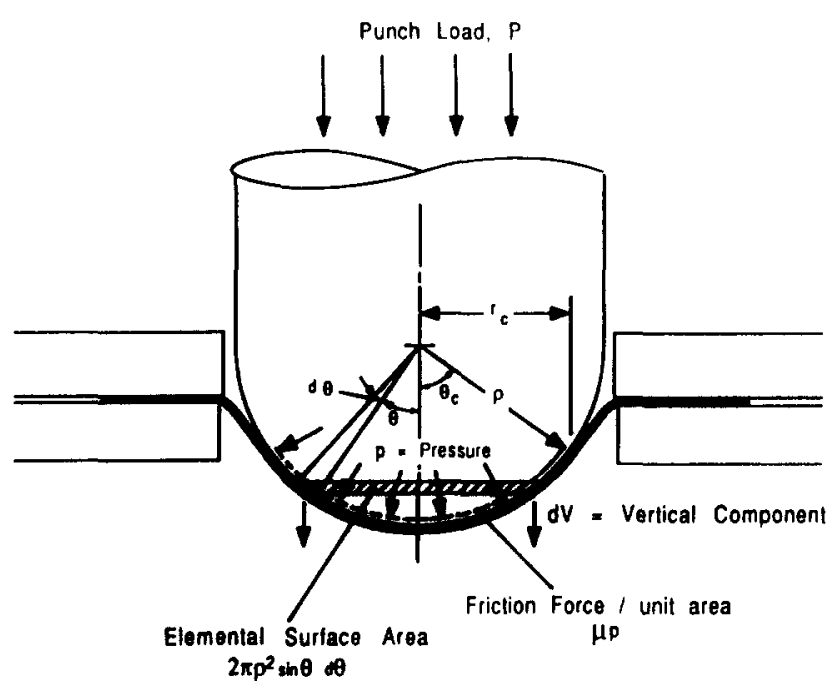

FIG. 3. A schematic representation of the sheet stretching process.

where $X=\left[\left(\mathrm{d} P / \mathrm{d} r_{\mathrm{c}}\right) / P\right]_{r_{0}}, r_{\mathrm{c}}=\rho \sin \theta_{\mathrm{c}}, r_{0}=\rho \sin \theta_{\mathrm{c}}$ and $\theta_{\mathrm{c}}=\theta_{0}$ at maximum pressure. The experimental load-deflection curves shown in Figs 5-7 have been used to evaluate $\mu$ in equation (8). The experimentally calculated overall coefficient of friction of each material at the punch-sheet interface is shown below:

for aluminum, $\mu=0.235$

for mild steel, $\mu=0.191$

for brass, $\mu=0.293$.

\section{THEORETICAL AND COMPUTATIONAL PROCEDURES}

The analyses presented here are based on the finite element program EPAFRIC [9], together with certain modifications to it which allow the solution of sheet stretching. The theoretical basis of the program is a rate formulation of isotropic elastic-plastic material behavior by employing the Jaumann stress rates to ensure material frame indifference. The onset of plastic yielding is characterized by the von Mises criterion and the Prandtl-Reuss equations. The program uses an incremental finite element analysis employing constant strain triangles.

One special feature of this program is the construction of an incremental constitutive relation for friction contact in analogy with the plasticity theory [10]. This feature accommodates appropriate variations in the interfacial conditions, such as contact pressure and the hardening or softening response in the transition from sticking to sliding motion. This is an attractive feature, because the broad goal of this research is to gain a better understanding of friction along the interface in sheet forming processes:

The original program has been modified to incorporate anisotropic properties of sheet metals and Green stress rates. The yield criterion proposed by Hill has been used to characterize the anisotropic effect [11]. It is known that the use of the most common objective stress rate, the Jaumann rate, in the generalization of the infinitesimal theory leads to an instability in the evolution of the yield surface which is explained on the basis of the elastic solution [12]. Any instability that appears in the elastic solution of the simple shear problem can also appear in the solution of an elasto-plastic problem in which kinematic hardening is used. In order to avoid this problem, Green stress rates [13] based on notions of invariance under superimposed rigid body motion have been adopted.

A material is said to be hypoelastic if the components of stress rate are linear functions of the components of the rate of deformation [14]:

$$
\sigma_{i j}^{\nabla}=c_{i j k l} D_{i j},
$$


where $\sigma_{i j}^{\nabla}$ represents any measure of the change of Cauchy stress that is unchanged apart from orientation under superposed rigid body motions. The rate of deformation tensor $D_{i j}$ is a well defined quantity; it vanishes when the body performs a rigid body motion. However, for a stressed body performing rigid body rotation, neither the time derivative $\partial \sigma_{i j} / \partial t$ nor the material derivative $\mathrm{D} \sigma_{i j} / \mathrm{D} t$ vanishes identically. A rigid body rotation changes the stress tensor, even though the state of stress in the body remains unchanged. Thus neither $\partial \sigma_{i j} / \partial t$ nor $\mathrm{D} \sigma_{i j} / \mathrm{D} t$ can serve as an appropriate stress rate measure to be related simply to the deformation rate $D_{i j}$. The stress rate tensor must contain a rotary term that compensates for the fact that the stress components with respect to a fixed coordinate system $x_{i}$ change, even when there is no change in the stress components with respect to a coordinate system $x_{j}^{\prime}$ that participates in the instantaneous rotation of the neighborhood of the considered particle. This is why a new rate derivative such as $\sigma_{i j}^{\nabla}$ is introduced. The desired stress rate derivative must be invariant with respect to rigid body rotation, but this requirement demands no unique answer.

Green's definition, denoted by $\sigma^{\nabla}$, is obtained by differentiating the expression for $\sigma$ in terms of $\sigma^{*}$, giving

$$
\sigma^{\nabla}=\dot{\sigma}+\sigma \Omega-\Omega \sigma=\mathbf{R} \sigma^{*} \mathbf{R}^{\mathrm{t}}
$$

where $\sigma^{*}$ is a rotated Cauchy stress, $\sigma^{*}=\mathbf{R}^{\mathrm{t}} \sigma \mathbf{R}, \Omega$ is a rate of rotation tensor, $\Omega=\dot{\mathbf{R}} \mathbf{R}^{\mathrm{t}}$, and $\mathbf{R}$ is an orthogonal rotation tensor that comes from the polar decomposition of the deformation gradient $\partial x_{i} / \partial X_{j}$. We see that this stress rate represents the derivative of an invariant measure of stress, $\sigma^{*}$, whose principal invariants have the same values as those for the Cauchy stress. Thus, if $\sigma^{\nabla}$ is zero, Prager's condition that the invariants of Cauchy stress be stationary is satisfied. The advantage of this rate measure over Jaumann's is that it is derivable from a physically meaningful stress, $\sigma^{*}$, and it is truly a measure of the rate of change of stress in the deformed configuration. This forms the theoretical basis for the finite element method.

Computations were carried out until punch depth reached $40 \mathrm{~mm}$ after 231 time increments. The punch speed was $1 \mathrm{~mm}$ per second and the data were obtained at each $5 \mathrm{~mm}$ punch travel. Mesh configurations are shown in Fig. 4 for each $5 \mathrm{~mm}$ punch travel. The total CPU time consumed for 527 nodes and 232 4-CST elements was about $20 \mathrm{~min}$. The program was run on an IBM 3090-400 computer.

\section{DISCUSSION OF RESULTS}

During the deformation process we assume that the interface pressure is uniform, that the coefficient of friction is constant and that the inflection point on the load-deflection plot corresponds to the attainment of maximum pressure. The average value of the coefficient of

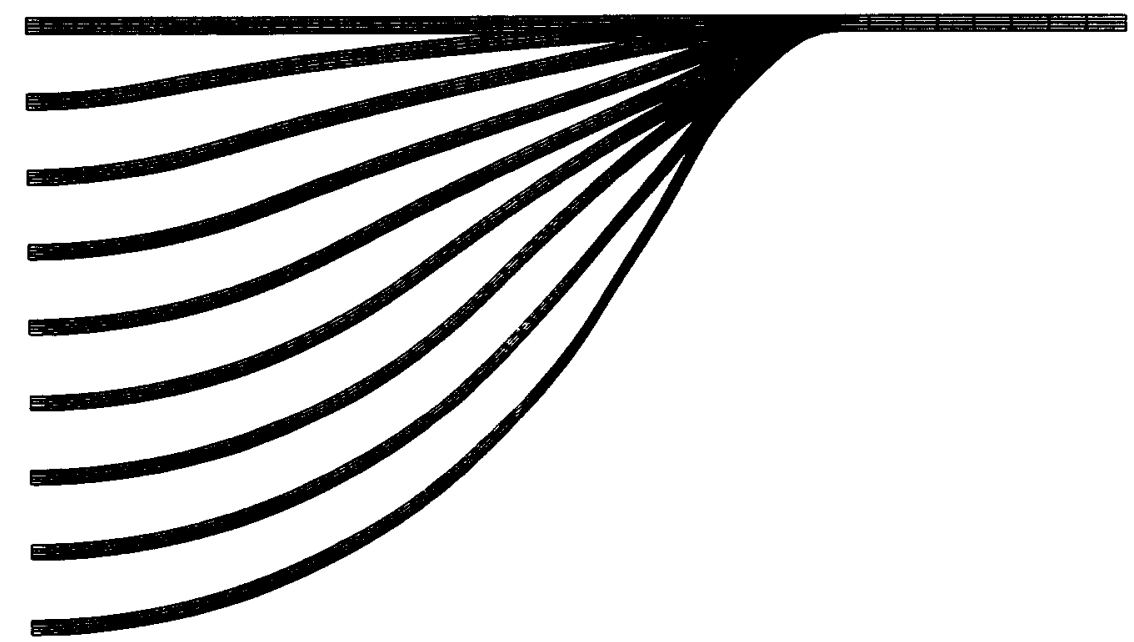

FIG. 4. Mesh configuration at different stages of sheet stretching. 
friction during stretching of thin sheets over a hemispherical punch was determined from the experiment under dry conditions. This experimentally determined coefficient of friction was used as one of the input data to the finite element code for theoretical analysis.

Many process parameters affect the value of the coefficient of friction along the punch -sheet interface. Those parameters are: surface roughness of punch and workpiece, lubricant, punch speed, contamination of surface. The coefficient of friction for $70: 30$ brass on steel under dry conditions was reported by Ghosh [8] as 0.36 . For steel on steel and aluminum on steel under dry conditions, the coefficient of friction value of 0.22 for both materials was also reported in the same paper. In our work, the measured coefficient of friction values for brass on steel, steel on steel and aluminum on steel were $0.293,0.191$ and 0.235 respectively. Although our present results cannot be compared directly with the research findings of Ghosh, because of different punch speeds and different classifications of materials, the trends are similar, especially for brass and steel. For example, a punch speed of $60 \mathrm{~mm} \mathrm{~min}^{-1}$ was used in our experiments, whereas Ghosh used $25.4 \mathrm{~mm} \mathrm{~min}^{-1}$. According to the classical work of Bowden and Tabor [15], the coefficient of friction decreases with increasing speed.

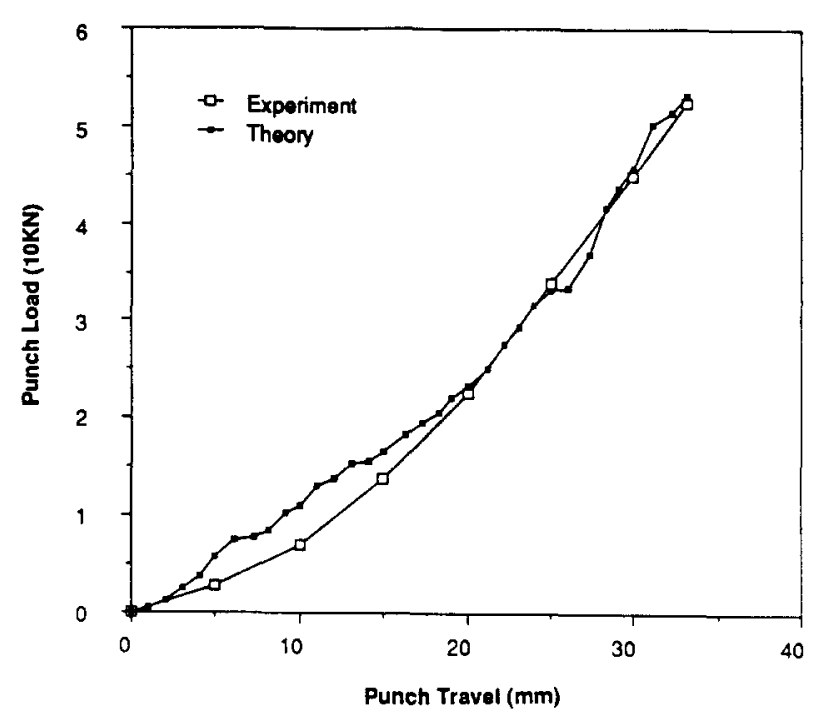

Fig. 5. Load-deflection curve for steel.

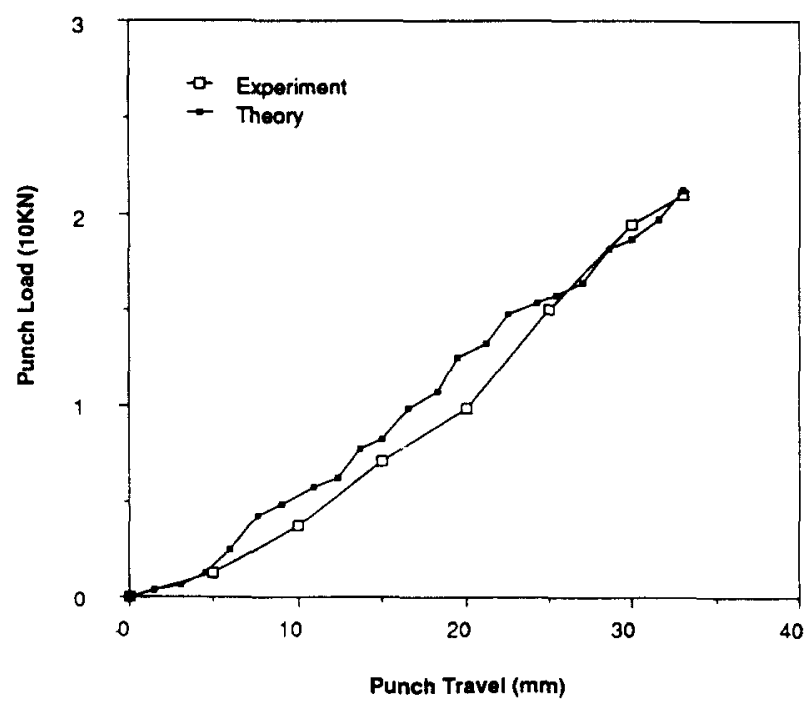

Fig. 6. Load-deflection curve for aluminum. 


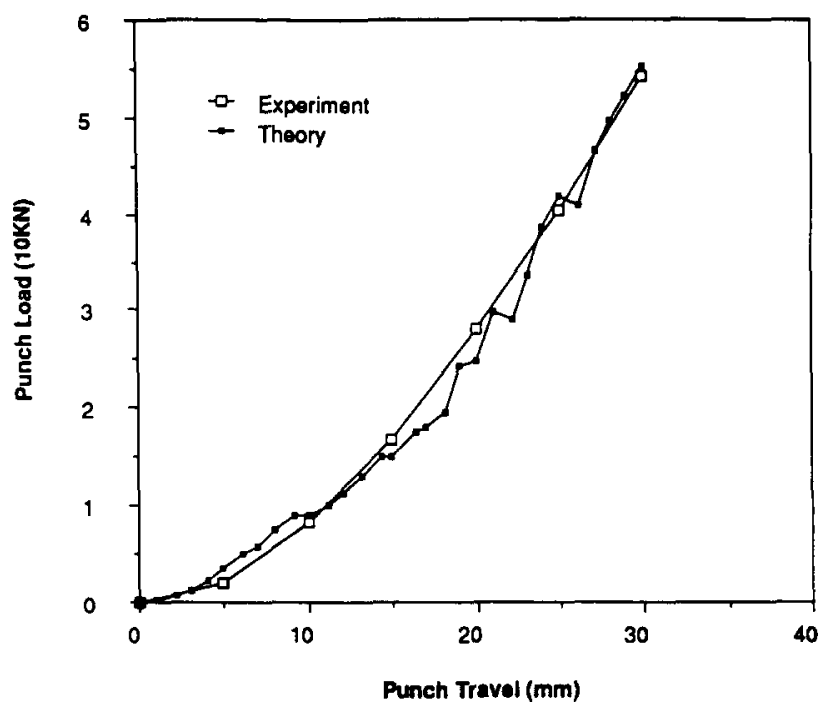

FIG. 7. Load-deflection curve for brass.

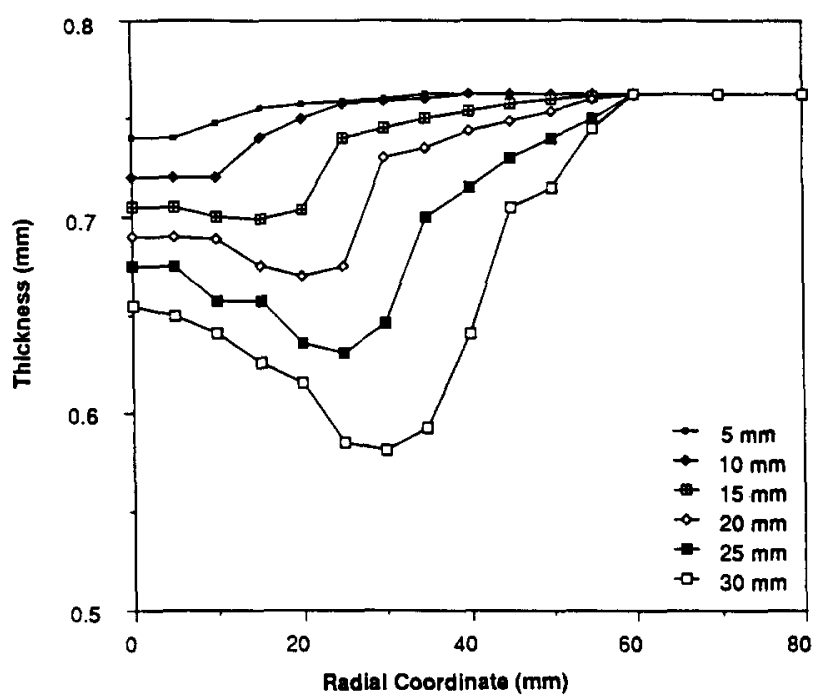

(a) Experiment

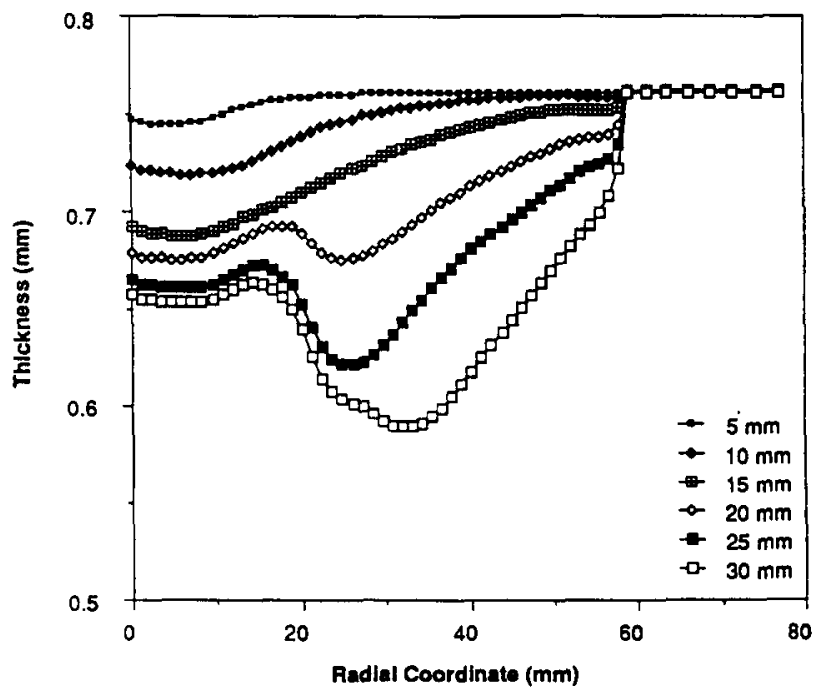

(b) Theory

FiG. 8. Radial thickness variation for steel. 
The overall coefficient of friction evaluated from experiments represents only an estimate of the average value. It is quite likely that the friction will change from point to point on the punch-sheet interface, and also as a function of deformation. Such variation would be difficult to determine, and if determined would change from one application to another.

Experimentally and theoretically obtained punch-load deflection plots for mild steel, aluminum and brass specimens are shown in Figs 5-7. Experimental and theoretical values are favorably matched. The small differences between experiment and theory shown in Figs 5-7 are mainly due to limited element numbers and stepwise contact conditions in numerical calculations. The maximum punch depth in this case is about $35 \mathrm{~mm}$.

The experimental measurements of thickness variations for each incremental stretching of mild steel, aluminum and brass specimens over a hemispherical punch are shown in Figures 8-10. As the punch travels, the friction forces increasingly retard the metal movement over the punch head, thus causing less deformation there and more in the unsupported areas. Thus the strain peak gradually shifts towards the edge of the hemispherical dome.

The thickness variations indicate that the stress and strain localization zones are severe at later stages in stretching. This implies that at a certain stage the sheet will have a surface depression or a neck. All specimens-steel, aluminum and brass-have been successfully



(a) Expariment

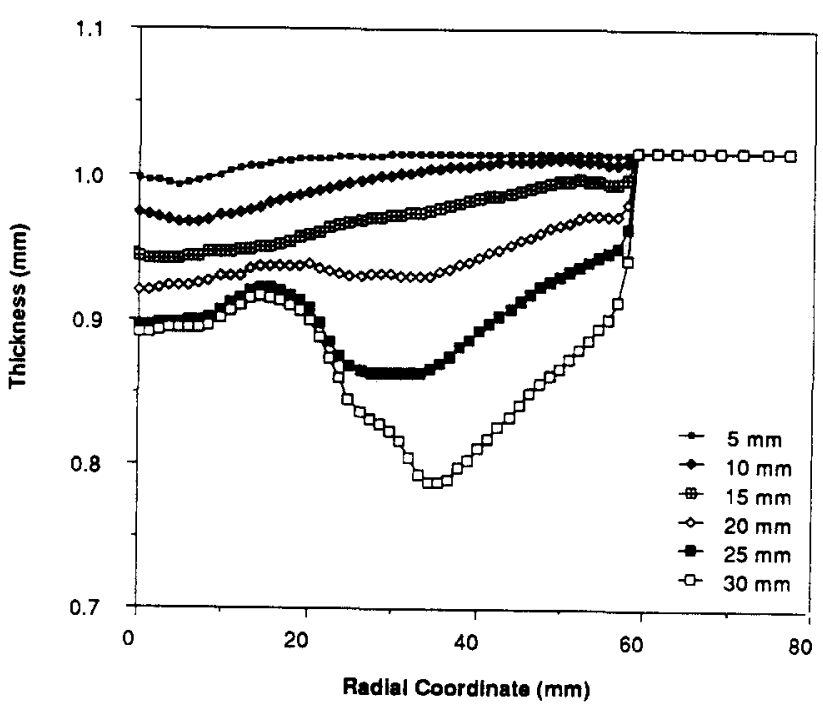

(b) Theory

Fig. 9. Radial thickness variation for aluminum. 


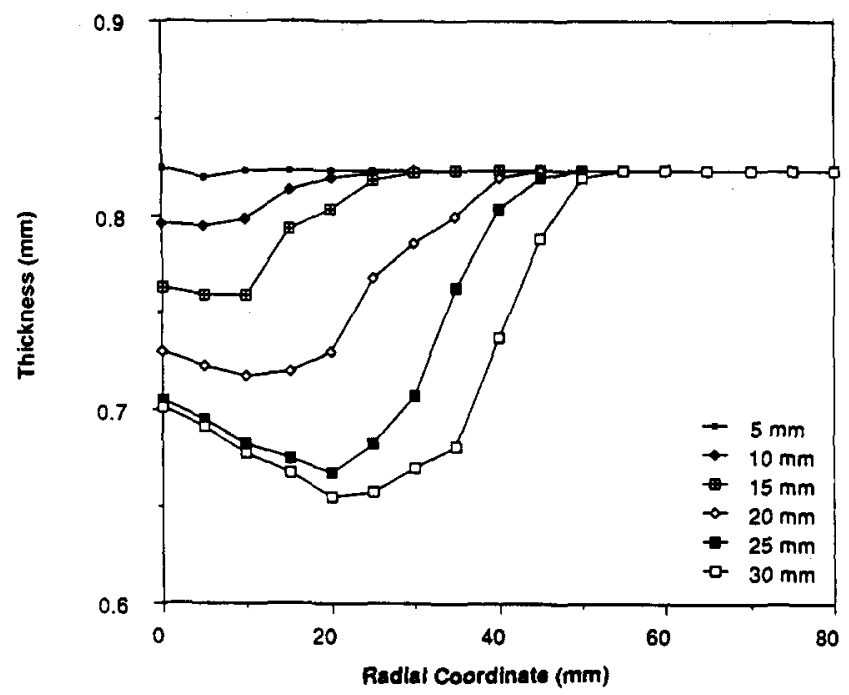

(a) Exporiment

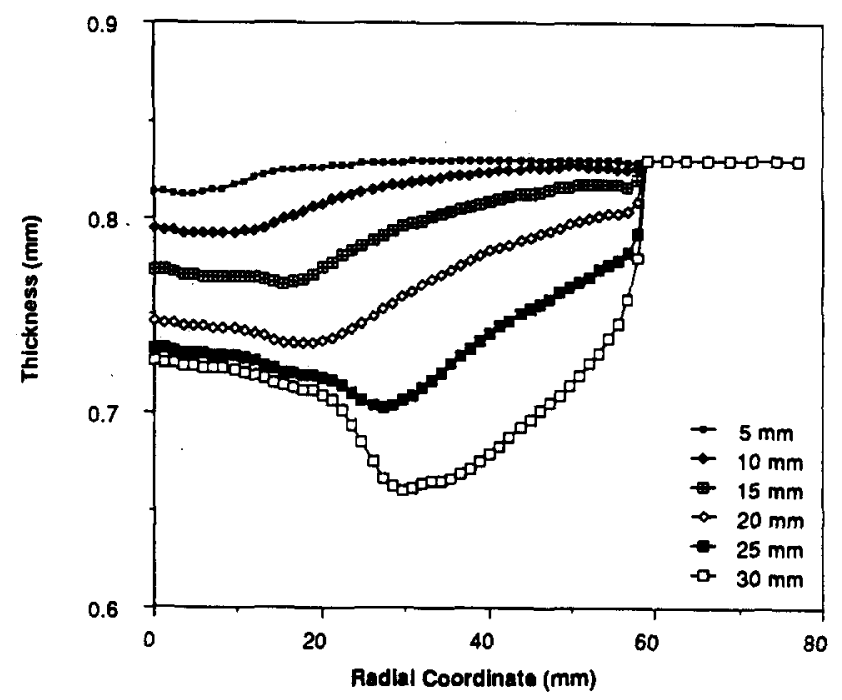

(b) Theory

FIG. 10. Radial thickness variation for brass.

stretched with no fracture until the final stage for $35 \mathrm{~mm}$ punch travel. Then we can expect that, as stretching proceeds, thinning at the root of the neck will be enhanced and will occur much faster than the deformation of the rest of the sheet, owing to the stress and strain localization. Initially, cold rolled aluminum sheet was used in our experiments, but its stretchability was limited by its prior cold work. Aluminum sheets were annealed to improve ductility. Hence the measured $\bar{r}$ values were close to unity.

From Figs 8-10 we can see that the overall shapes are well matched. However, the contact regions along the punch-sheet and die-sheet interfaces are not well matched. This suggests that our experimentally calculated overall coefficient of friction is not good enough to model the real forming processes. It seems that the actual value of the coefficient of friction along the punch-sheet interface is somewhat less than the prescribed value in the analysis as obtained from experiments. This situation is reversed in the contact region along the die-sheet interface. This means that the coefficient of friction varies from point to point, and we need a better understanding of such local variations in friction along the punch-sheet interface.

Although the theoretical results compare favorably with the experimental results, it has been realized that a more realistic frictional variation along the punch-sheet interface is needed. In fact, research is in progress to investigate such frictional boundary conditions 
using a new transparent punch technique. In this experimental technique, the asperities of sheet metal along the punch-sheet interface are observed and recorded. A new friction model will be developed on the basis of the microscopic asperity analysis of the punch-sheet interface. Such accurate description of frictional variations along the interface will further enhance the predictive capabilities of the numerical methods.

\section{CONCLUSIONS}

Experiments and theoretical computations on sheet stretching have been performed and the results compared. Experiments have been conducted on commercial materials such as mild steel, aluminum and brass with a hemispherical steel punch under dry conditions. The theoretical computations employed a large-strain finite element technique based on an elastic/work-hardening-plastic material model incorporating anisotropic properties. A special feature of this research is the linking of the analysis to the experiment by determining the overall coefficient of friction along the punch-sheet interface. Such measured coefficients of friction have been used as inputs to the numerical analysis. In this way, very good agreement has been achieved between experimental and theoretical results such as loads, deflections and strain distributions at various stages of the sheet stretching process. Finally, research is in progress to refine the frictional boundary conditions further by actually developing a new friction model based on the microscopic asperity analysis.

\section{REFERENCES}

1. S. Y. Chung and M. W. Swift, Cup-drawing from a flat blank. Proc. Inst. Mech. Engng 165, 199 (1951).

2. N. M. WANG and B. BUdiansky, Analysis of sheet metal stamping by a finite element method. J. appl. Mech. Trans. ASME 45, 73 (1978).

3. N. M. WANG and M. L. WENNER, Elastic-viscoplastic analysis of simple stretch forming problems. In Mechanics of Sheet Metal Forming, Symposium held at the General Moters Research Laboratories, Warren, Michigan, p. 367 (1977).

4. C. H. LEE and S. KoBayaSHI, New solutions to rigid-plastic deformation problems using a matrix method. $J$. Engng Ind. Trans. ASME 95, 865 (1973).

5. S. Kobayashi and J. H. KIM, Deformation analysis of axisymmetric sheet metal forming processes by the rigid-plastic finite element method. In Mechanics of Sheet Metal Forming, Symposium held at the General Moters Research Laboratories, Warren, Michigan, p. 345 (1977).

6. B. B. YOON, R. S. RAO and N. KIKUCHI, Experimental and numerical comparisons of sheet stretching. In Proc. 16th N. Am. Manufacturing Res. Conf., University of Illinois, Urbana, p. 159 (1988).

7. R. S. RAO and S. D. K. BARbat, Frictional interactions in forming processes: interfacial observations and photoelastic studies with transparent dies. Proc. Manufacturing Processes, Machines and Systems, 14th Conf. on Prod. Res. and Tech. held at The University of Michigan, p. 105 (1987).

8. A. K. GHosh, A method for determining the coefficient of friction in punch stretching of sheet metals. Int. $J$. Mech. Sci. 19, 457 (1977).

9. J.-H. ChENG and N. KIKUCHI, An analysis of metal forming processes using large deformation elastic plastic formulations. Comput. Meth. appl. Mech. Engng 49, 71 (1984).

10. J.-H. Cheng and N. KIKUCHI, An incremental constitutive relation of unilateral contact friction for large deformation analysis. J. appl. Mech. Trans. ASME 52, 639 (1985).

11. R. Hill, The Mathematical Theory of Plasticity. Oxford University Press, London (1950).

12. G. C. Johnson and D. J. BAUMman, A discussion of stress rates in finite deformation problems. Sandia Report Sand 82-8821 (1982).

13. A. E. GREen and P. M. NAGHDi, A general theory of an elastic-plastic continuum. Arch. Rat. Mech. Anal. 18. 251 (1965).

14. W. Prager, Introduction to Mechanics of Continua. Ginn and Company, Boston (1961).

15. F. P. Bowden and D. TABor, The Friction and Lubrication of Solids, part II. Oxford University Press, London (1964). 